Running Head: PRIOR VERBAL DESCRIPTION

Prior Verbal Description of Novel Stimuli Improves the Learning of Information Integration Categories but not Rule-Described Categories

\author{
Sarah J. Miles \\ Bailey Brashears \\ Joshua Hatherley \\ John Paul Minda
}

Department of Psychology

The University of Western Ontario

John Paul Minda, PhD

Department of Psychology \&

Brain and Mind Institute

The University of Western Ontario

London, ON N6A 5C2 CANADA

jpminda@uwo.ca

Word Count: 5708 
A prominent theory of category learning assumes that people rely on two parallel and competing systems that make use of either the abstraction of verbal rules (explicit system) or the gradual association of the category exemplars with the appropriate response (implicit system). Because the explicit system relies on verbal processing, we hypothesized that priming the verbal system by asking participants to provide a verbal description of some of the stimuli prior to classification would enhance the learning of rule-described categories but would have no effect on the learning of information integration categories. Our results failed to confirm the hypothesis, and we observed the opposite pattern: prior verbal description enhanced learning of the information integration categories but not the rule-described categories. Our data and subsequent modelling suggest that participants in both categories tended to rely on a rule-based strategy, but participants were quicker to abandon that strategy when they had prior exposure to the stimuli. 


\section{Prior Verbal Description of Novel Stimuli Improves the Learning of Information Integration Categories but not Rule-Described Categories}

A common theme across many domains of cognition is the existence of multiple cognitive systems dedicated to solving a single task. For example, reasoning can be carried out using heuristics or rules (Evans, 2003; Sloman, 1996), attitudes can be formed through implicit or explicit processes (Gawronski \& Bodenhausen, 2006), and memories can be supported by declarative or nondeclarative networks (Eichenbaum, 1997). The interaction between the systems is an important aspect of any multiple systems theory.

Within the domain of category learning a class of theories suggests that categorization decisions can be made using a verbal or a nonverbal system (Ashby, Alfonso-Reese, Turken, \& Waldron, 1998; Minda \& Miles, 2010; Smith \& Grossman, 2008). The verbal system learns rule-described (RD) categories for which category membership can be determined by applying a simple verbal rule (e.g., mammals are animals that possess fur or hair). This type of learning involves active hypothesis testing in order to identify and apply the optimal categorization rule. Working memory and executive functions (i.e., cognitive abilities used to guide effortful behaviour) operate to identify stimulus dimensions on which a rule could be applied, to generate and apply a rule, to process feedback, to decide whether to switch to a new rule, and to remember which rules have already been tested (Miles \& Minda, 2011; Waldron \& Ashby, 2001; Zeithamova \& Maddox, 2006, 2007). Categories based on a single, salient dimension are easiest for the verbal system to learn while categories based on a less salient dimension, 
multiple dimensions, or complex rules are more difficult for the verbal system to learn (Filoteo, Maddox, Ing, \& Song, 2007; Maddox, Filoteo, \& Lauritzen, 2007; Nosofsky, Stanton, \& Zaki, 2005; Zeithamova \& Maddox, 2007).

The nonverbal system can learn categories that are not defined by a rule (non-rule-described or NRD). This includes information-integration (II) categories, which are non-rule-described categories for which category membership is based on multiple dimensions that must be integrated before the categorization decision can be made (e.g., dogs are animals that can sometimes bark, sometimes have fur, sometimes have a tail, etc.). This system learns categories implicitly and relies on procedural learning mechanisms (Ashby, Maddox, \& Bohil, 2002; Maddox, Bohil, \& Ing, 2004) and dopamine-mediated feedback processing (Maddox, Ashby, \& Bohil, 2003; Maddox \& Ing, 2005). Active cognitive processes like executive functioning and verbal working memory are not thought to play a role in learning once the nonverbal system is engaged.

The two systems work in parallel, but the verbal system is often thought to be the default system. People tend to approach new categorization tasks by attempting to find a rule, by bringing verbal processes to bear on the problem of learning which features are predictive of category membership, and by hypothesis testing potential categorization rules. If this verbal-based strategy is successful, participants will abstract and store a rule and continue to refine the rule-based approach and apply the rule to novel stimuli. If the verbal strategy is not successful (i.e. if responding on the basis of the verbal rule does not generate enough correct answers), learners may transition to the nonverbal system (Ashby et al., 1998; Ashby \& Valentin, 2017; Minda \& Miles, 2010). But even for 
non-rule-described categories, participants may begin with a rule-based approach and only transition to the non-rule-described approach after exhausting all the potential rules, and only after the stimulus response associations have had enough time to accumulate. Mathematical modeling of participants' categorization strategies during II learning shows that they tend to begin the task using RD strategies but switch to II strategies as learning progresses (e.g., (Maddox, Filoteo, Hejl, \& Ing, 2004; Markman, Maddox, \& Worthy, 2006; Worthy, Maddox, \& Markman, 2009).

\section{Exposure to the stimuli before learning}

Because the verbal system is often the default approach that people take when learning new categories, any prior intervention that facilitates or emphasises the verbal description of the stimuli should also facilitate RD category learning. Several studies from my lab have found evidence linking working memory capacity with the ability to learn RD categories but not with the ability to learn NRD categories. Although we are attempting to keep self citations to a respectable level, a few of these studies bear directly on the present work. The most relevant are three series of experiments that examined how young children differ from young adults when learning RD and NRD categories and also how older adults differ from young adults when learning these kinds of categories. With respect to young children, we found that children and adults learned single-dimensional $\mathrm{RD}$ categories and NRD family-resemblance categories in the same way but that younger children showed greatly diminished learning relative to adults when learning categories that required the abstraction of a more complex XOR rule (Minda, Desroches, \& Church, 2008). Of particular relevance to the present work is that 
the second experiment found that requiring adults to learn the same categories while performing a coarticulation task that interfered with verbal working memory reduced adults' performance, effectively making the adults learn the XOR categories like children. And a third experiment in which children and adults were first asked to verbally describe each exemplar prior to learning improved the performance of both groups but also enabled the children to learn the complex XOR rules at adult-like levels.

More recent research found a strikingly similar pattern when comparing category learning performance of healthy older adults with healthy younger adults on the same category sets that were used by Minda et al above (Rabi \& Minda, 2016). While older adults performed just as well as younger adults on single-dimensional RD categories and family-resemblance NRD categories, they failed completely to learn the more complicated XOR rule categories. We noted again the relationship with verbal working memory. A subsequent study then employed the technique of exposing participants to a pretraining task in which they viewed and described the exemplars prior to learning (Rabi \& Minda, 2017). We found that this pretraining task improved the ability of older and younger adults when learning the XOR RD categories and also enabled older adults to improve when learning other kinds of $\mathrm{RD}$ categories composed of continuously varying stimuli. In all of these studies, we speculated that the pretraining task reduced the cognitive demands of the category learning task by making clear how many categories there were, by making clear what features mattered in the task, and by making clear that exemplars could be grouped together according to these features. 
Although our research found that the pretraining task improved performance on the categories that placed the strongest demands on working memory and executive functions (i.e. the XOR RD task) we did not arrive at a clear explanation as to how and why the effect happened. A caveat to this work is that the pretraining task was multifaceted. Participants viewed each exemplar that they would later learn to classify, they were asked to name each one of the features, and they then saw the entire set of exemplars on the screen at once. Although the verbal naming process likely had an effect on the subsequent encoding and learning, seeing all of the exemplars together likely also had a strong effect of how participants came to understand the task. In addition, participants were also naming and describing the same stimuli that they would later learn to classify, which could have assisted learning in general as a result of familiarity.

The pretraining task was designed like this because we wanted to demonstrate in principle that young children and older adults could learn a category that required the use of an XOR rule. But the design of this task did not make clear which of these facets was contributing to the effect. We do not know if it was the verbal description that enabled participants to learn the complex rules, if it was the act of viewing all of the exemplars that enabled them to learn the complex rules, or if it was a combination of both. The present work was designed to test the hypothesis that asking participants to verbally describe stimuli prior to learning the same kinds of stimuli will facilitate learning RD categories but not II categories. We make this prediction because the verbal description of stimuli should reduce the working memory demands of the early phases 
of the rule learning process by making clear what the constituent features are and how to verbalize them.

It stands to reason that the ability to describe a set of stimuli relies heavily on active cognitive processes like executive functioning. When presented with series of stimuli that vary on several dimensions, participants that are asked to verbally describe those stimuli should show better performance on subsequent category learning than non-describers. However, as this relies heavily on verbal working memory, this improved performance should be targeted exclusively on RD categories, which rely on executive function and verbal categorization. The same description task should not benefit the learning of II categories because these categories are thought to be learned via procedural mechanisms and the verbal description of each stimulus dimension should not confer any specific advantage. Because the explicit system relies on verbal processing, it is hypothesized that priming the verbal system by asking participants to provide a verbal description of some of the stimuli prior to classification should enhance learning of rule-described categories but will have no effect on the learning of information integration categories.

\section{The Current Study}

Our experiment manipulates the efficiency of hypothesis testing via stimulus knowledge and measures its subsequent effect on performance in learning RD categories. Prior to categorization, participants will either describe a selection of stimuli like the ones they will learn to classify or a set of completely unrelated stimuli. The stimuli they describe are not presented in a way that indicates which category they will 
be assigned to. We hypothesize that the stimulus description will familiarize participants with important aspects of the stimuli, including number and range of stimulus dimensions. This knowledge is expected to help participants who learn RD categories proceed efficiently through the hypothesis testing procedure.

\section{Method}

\section{Participants}

Participants included 123 undergraduate students with a mean age of 18.22 years $(S D=0.86$ years $)$ from the University of Western Ontario who participated in the study for research credits. Sixty-one participants learned the RD category (30 in the Control condition, 31 in the Describe condition) and sixty-two learned the II category (32 in the Control condition, 30 in the Describe condition).

\section{Materials}

The categorization stimuli were sine-wave gratings (pictured in Figure 1) that varied in spatial frequency and spatial orientation. The categorization rule for the RD category set is based on the frequency of the alternating light and dark bands, such that gratings with fewer lines go into Category A and gratings with many lines go into

Category B. In Figure 1A, the vertical line separating Category A and Category B, known as the decision bound, represents the strategy that maximizes categorization accuracy (Ashby \& Gott, 1988). The NRD category in this study was an Information Integration (II) category (Figure 1B) in which both frequency and orientation need to be integrated before the categorization decision is made. The decision boundary in Figure $1 \mathrm{~B}$ can be expressed as "If the lines in the grating have a smaller orientation than frequency, the 
grating goes in Category A; otherwise, it goes in Category B.” However, this is not a practicable categorization rule because frequency and orientation are not directly comparable; instead, this category is thought to be learned procedurally.

A. Rule-defined

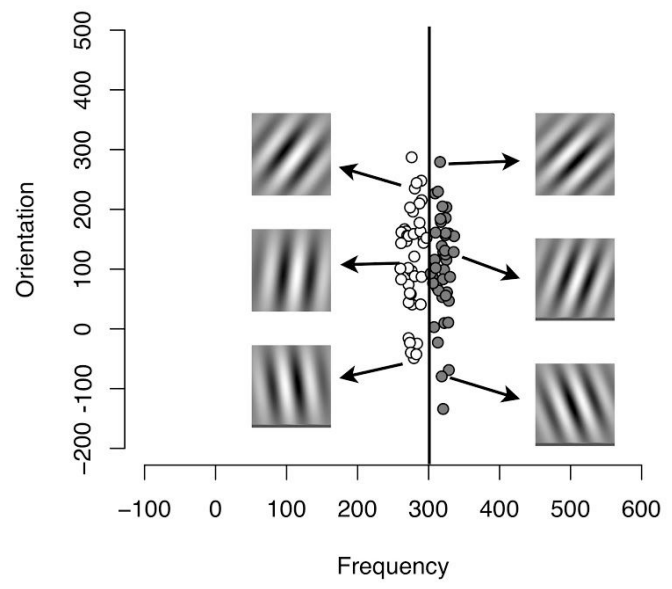

B. Information Integration

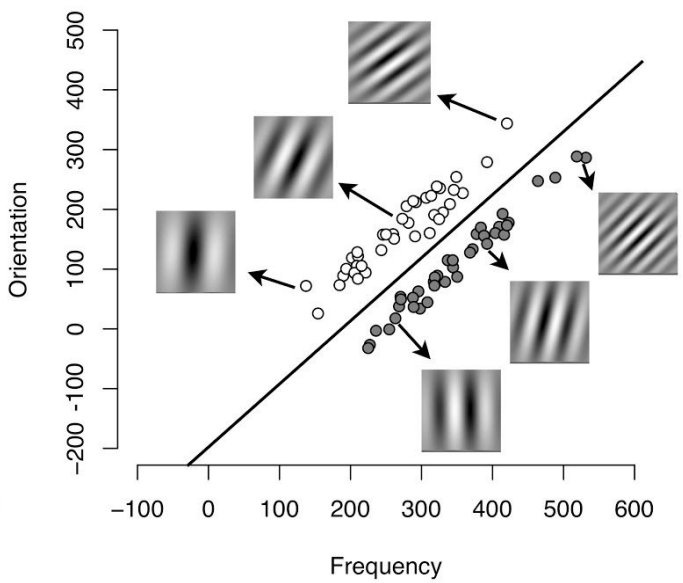

Figure 1. A) The Rule-Defined (RD) category structure used in the experiment. Each light circle represents a stimulus from Category A and each dark circle represents a stimulus from Category. Also shown are three representative exemplars from each category B) The Information Integration (II) category structure.

For the RD category set, 80 stimuli were generated (see (Ashby \& Gott, 1988) for more details), with 40 in Category A and 40 in Category B. The distribution of each category was specified by a mean and variance for frequency and orientation, and covariance between them as shown in Table 1 . Stimuli for each category were generated by randomly sampling 40 coordinates from the appropriate multivariate normal distribution and using the GRT package in R. (R Core Team \& Others, 2008) to generate a sine-wave grating corresponding to each coordinate. Sine-wave grating frequency was calculated as $f=.25+\left(\frac{x_{f}}{50}\right)$ cycles per gradient and orientation was calculated 
$o=x_{o}\left(\frac{\pi}{500}\right)$. Stimuli for the II category set were generated in the same way except that different parameter values were used (see Table 1). The resulting category structures for the RD and II category sets are illustrated in Figure 1A and 1B. Stimulus parameters and generation were the same as those used by (Zeithamova \& Maddox, 2006) and (Miles \& Minda, 2011).

Table 1: Distribution Parameters for the Category Sets

\begin{tabular}{lccccc}
\hline Category Structure & $\mu \mathrm{f}$ & $\mu \mathrm{o}$ & $\sigma 2 \mathrm{f}$ & $\sigma 20$ & $\operatorname{cov}$ f,o \\
\hline Rule-described & & & & & \\
$\quad$ Category A & 280 & 125 & 75 & 9000 & $\mathrm{o}$ \\
$\quad$ Category B & 329 & 125 & 75 & 9000 & 0 \\
$\quad \begin{array}{l}\text { Information Integration } \\
\quad \text { Category A }\end{array}$ & 268 & 157 & 4538 & 4538 & 4351 \\
$\quad$ Category B & 332 & 93 & 4538 & 4538 & 4351 \\
\hline
\end{tabular}

Notes. $\mathrm{f}=$ frequency, $\mathrm{o}=$ orientation for the sine-wave gratings

The actual stimuli were $370 \times 370$ pixels in size and partially masked with a circle and two solid rectangles at the base so that each stimulus resembled a crystal ball (see Figure 2A for examples). The original stimuli stimuli can be found at

\section{https://osf.io/4kap3/.}

\section{Procedure}

Participants were tested one at a time in the lead author's research lab. Prior to entering the lab, participants were randomly assigned to one of the experimental conditions (Describe or Control) and one of the two category sets (Rule-Described or Information Integration). Upon entering the lab, each participant provided written 
consent, was seated at a desk with a computer, headphones, and a blank experiment booklet. The were asked to first read the instructions on the sheet and was first asked to read the instructions on the screen. The experimenter left the room and the experiment began.

\section{A. Crystal Ball Stimuli}
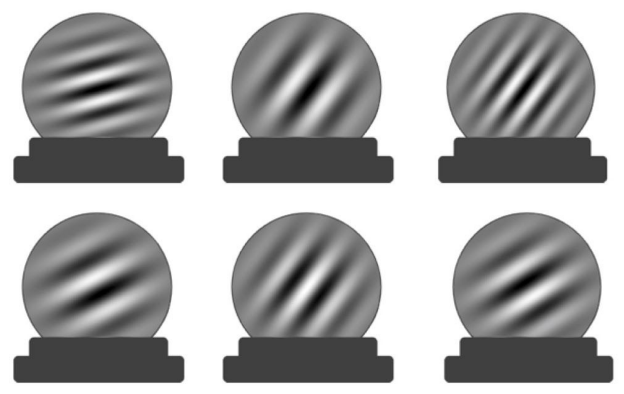

\section{B. Cartoon Face Stimuli}



Figure 2. The stimuli used in the description task. Participants in the Describe condition saw each of the six crystal balls shown in Panel A. Participants in the Control condition were shown each one of the cartoon faces in Panel B.

After reading the instructions, participants began the picture description task.

Participants in the Describe condition were shown six crystal ball sine-wave gratings with varying frequency and orientation (see Figure $2 \mathrm{~A}$ for examples), one at a time. Participants were given 60 seconds to provide a written description of each one, using 
the blank experiment booklet to write their descriptions. At the end of each 6o-second period, participants heard a beep in their headphones indicating that a new crystal ball had been presented on the screen and that a new description should be written. The six crystal ball stimuli were chosen to have a range of frequency and orientation values, but this range did not provide any information about the subsequent category learning task. The control condition followed the same procedure except that participants described cartoon faces (see Figure 2B for examples) rather than crystal balls. The faces varied across two dimensions (mouth width and eye height) so that participants in both conditions described stimuli with two varying dimensions.

Following the picture description task, participants then began the category learning task. Participants learned either a RD category or an II category according to which condition they were assigned. On each trial, participants saw a single sine-wave grating and assigned it to either Category A or Category B by pushing the button labeled A (1 key) or B (o key), respectively. The word correct or incorrect was displayed for 1500 ms followed by a $1000 \mathrm{~ms}$ inter-trial interval. The categorization stimulus remained on the screen throughout categorization and feedback.

\section{Results}

All of the data and the analysis code for this experiment are available at https://osf.io/4kap3/.

\section{Stimulus Description}

For each participant, the number of varying features (i.e., frequency and orientation for crystal balls; eye height and mouth width for faces) mentioned across all 
six written descriptions were counted (o, 1 or 2$)$. On average, participants who described faces in the Control condition mentioned about the same number of features $(M=1.73$, $\mathrm{SD}=0.55)$ as participants who described crystal balls in the Describe condition $(\mathrm{M}=$ 1.77, $\mathrm{SD}=0.46), t(121)=0.49, p=.63$.

\section{Categorization Performance}

Categorization performance (i.e. proportion correct) was calculated at each block in each condition and each category set by averaging across participants. The resulting $\mathrm{RD}$ and II learning curves are presented in Figure 3. Our primary hypothesis sought to examine the influence that exposure and description of stimuli prior to testing would have on later hypothesis testing in RD categories. A 2 (Category) x 2 (Condition) x 4 (Block) ANOVA was conducted, with category and condition as between subject factors and block as a within subject factor. Results indicated that a main effect of block occurred, indicating that learning developed across the blocks, $F(3,357)=51.79, p<$ $0.001, \eta^{2}=.30$. Additionally, a main effect of category was seen, suggesting that performance on RD categories was higher than performance on II categories, $F(1,119)$ $=38.04, p<0.001, \eta^{2}=.24$. Finally, we observed a main effect of condition, indicating that performance was improved for participants in the Describe condition, when compared to the Control condition, $F(1,119)=6.11, p=0.015, \eta^{2}=.05$. Our primary prediction was that the Describe/Control condition would improve performance on $\mathrm{RD}$ categories, but not II categories. The test of this hypothesis was a two way interaction between category and condition. This interaction was not significant, $F(1,119)=0.20, p$ $=0.652, \eta^{2}<0.01$. 
The main effect of Condition suggested that performance improved in both $\mathrm{RD}$ and II categories. Despite the lack of predicted interaction effect, Figure 3 suggests that the Describe condition did not seem to improve performance for participants learning RD categories, but it did improve performance on II categories.


Figure 3. The mean performance for participants learning each category set grouped by block and separated by condition. Panel A shows the mean performance of participants learning Rule Described Categories grouped by block and separated by condition. Panel B shows the mean performance of participants learning Information Integration Categories. The error bars denote SEM.

We did not predict this effect. We conducted two exploratory analysis in order to probe these unexpected patterns. An exploratory hypothesis was proposed; that the 
Describe condition did not improve performance for participants learning $\mathrm{RD}$ categories, but did improve the performance of participants learning II categories. We tested this hypothesis by conducting a 2 (condition) x 4 (block) ANOVA for each category set (RD and II). Because two related hypothesis were tested, we used a Bonferroni correction to the alpha level with $0.05 / 2=0.025$.

A 2 (condition) x 4 (block) mixed ANOVA was carried out on RD categorization performance and revealed a main effect for block, indicating that participants learned across the experiment, $F(3,177)=30.47, p<.001, \eta^{2}=0.34$. There was no main effect of condition $\left(F(1,59)=1.53, p=.22, \eta^{2}=0.03\right)$ nor interaction between block and condition $\left(F(3,177)=0.05, p=.99, \eta^{2}=0.01\right)$, indicating that $\mathrm{RD}$ performance in the Describe group was not significantly different from the performance of the Control group.

A second 2 (condition) x 4 (block) mixed ANOVA was carried out for II performance. Again, there was an effect of block, $F(3,180)=21.44, p<.001, \eta^{2}=0.26$. There was no interaction between block and condition, $F(3,180)=1.59, p=.201, \eta^{2}=$ o.03. However, a significant main effect of condition was found, confirming that performance of the Describe group was significantly higher than performance of the Control group in II, $F(1,60)=6.28, p=.015, \eta^{2}=0.09$.

\section{Categorization Strategy}

To investigate the strategy used by participants in each condition, a number of decision-bound models based on the General Recognition Theory (GRT) were fit to each participant's categorization responses at each block (Ashby \& Gott, 1988; Maddox \& 
Ashby, 1993). According to GRT, a categorization strategy can be represented as a decision boundary that splits multidimensional perceptual space into categories (e.g., Category A, Category B) with associated responses (e.g., push the A key, push the B key). Each stimulus is represented as a point in perceptual space, and the corresponding categorization response is generated by determining the response region into which the stimulus falls (Ashby \& Gott, 1988). Essentially, these models work by comparing the response a participant would have given had they used a particular strategy with the response they actually gave. The model is said to fit the participant's data to the extent that the model's predicted responses correspond with the participant's actual responses.

Four models were fit to each participant's pattern of responses, separately for each block: (1) a frequency rule model (optimal for the RD categories), (2) an orientation rule model, (3) an information integration model (the optimal model for II categories), and (4) a random guess model. (see the Appendix of the current paper and the OSF page for more information on how these models were fit https://osf.io/4kap3/). For every participant, at every block, the class of the best-fitting strategy (i.e., the one with the lowest AIC) was noted. The proportion of participants using an optimal strategy across blocks, description condition and category sets can be seen in Table 2 . 
Table 2: Number of Participants Fit By Each Model

\begin{tabular}{|c|c|c|c|c|}
\hline \multirow[b]{2}{*}{ Block } & \multicolumn{4}{|c|}{ Strategy } \\
\hline & $\begin{array}{l}\text { Information } \\
\text { Integration }\end{array}$ & Frequency & Orientation & Guess \\
\hline \multicolumn{5}{|c|}{ RD Control (N-30) } \\
\hline 1 & o & 22 & 1 & 7 \\
\hline 2 & 2 & 24 & O & 4 \\
\hline 3 & 2 & 26 & $\mathrm{O}$ & 2 \\
\hline 4 & o & $\mathbf{2 8}$ & $\mathrm{O}$ & 2 \\
\hline \multicolumn{5}{|c|}{ RD Describe $(\mathrm{N}=31)$} \\
\hline 1 & o & $\mathbf{2 3}$ & $\mathrm{O}$ & 8 \\
\hline 2 & 1 & $\mathbf{2 7}$ & O & 3 \\
\hline 3 & 1 & 29 & $\mathrm{O}$ & 1 \\
\hline 4 & 1 & $\mathbf{3 0}$ & $\mathrm{O}$ & $\mathrm{O}$ \\
\hline \multicolumn{5}{|c|}{ II Control $(\mathrm{N}=32)$} \\
\hline 1 & 4 & 20 & o & 8 \\
\hline 2 & 5 & 24 & o & 3 \\
\hline 3 & 5 & 25 & $\mathrm{O}$ & 2 \\
\hline 4 & 5 & 24 & $\mathrm{O}$ & 3 \\
\hline \multicolumn{5}{|c|}{ II Describe $\mathrm{N}=30$ ) } \\
\hline 1 & 4 & 22 & $\mathrm{O}$ & 4 \\
\hline 2 & 9 & 21 & $\mathrm{O}$ & $\mathrm{O}$ \\
\hline 3 & $\mathbf{1 1}$ & 19 & O & O \\
\hline 4 & 11 & 19 & O & O \\
\hline
\end{tabular}

Note: Optimal model is shown in bold.

By far, the dominant approach to across all conditions and categories was the frequency model. This was the optimal model for participants learning the RD categories and there was little evidence that the prior verbal description task had any 
effect of the adoption of that strategy. The was also the most common approach for participants who were learning the II categories, despite being a suboptimal strategy. However, participants who first engaged in a verbal description task were more likely to switch to the optimal information integration strategy as the learning session progressed.

We were interested in testing whether or not the verbal description task had an effect on optimal strategy. We calculated the proportion of participants in each condition and block that were best fit by the optimal model for the category set that participants were learning. The resulting proportions are shown in Figure 4 and shows that participants who learned the RD categories were generally fit best by the frequency model, irrespective of condition. For the participants who learned the II categories, however, the proportion of of the sample who were fit best by the optimal II model was higher for participants who were in the Describe condition. We tested this effect by fitting a logistic regression with condition and block predicting the dependant variable of whether or not a participant block was fit best by the optimal model or not. 

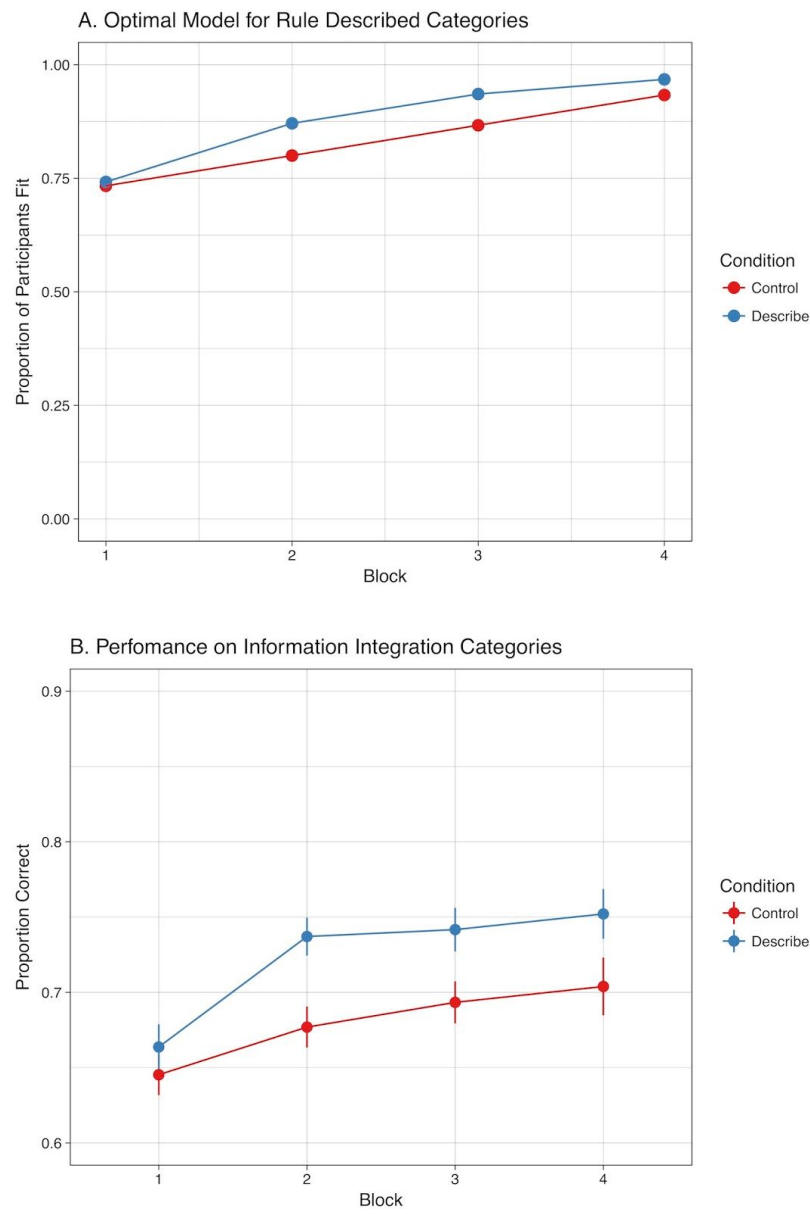

Figure 4. The mean performance for participants learning each category set grouped by block and separated by condition. Panel A shows the mean performance of participants learning Rule-Described Categories grouped by block and separated by condition. Panel B shows the mean performance of participants learning Information Integration Categories. The error bars denote SEM

The results of the regression are shown in Table 3. The resulting regression model was then tested with Chi-Square test of deviance with ANOVA against a model that included only the intercept. Because we are conducting two analyses, one for RD categories and one for II categories, we adopt the Bonferroni corrected alpha of $p=.025$ 
for significance. In this case, a significant result is taken as evidence that a given predictor accounts for the deviance from a null model with no additional predictors.

Table 3: Logistic Regression on Optimal Model Fits

\begin{tabular}{lcccc}
\hline RD Categories & $\mathrm{b}$ & $\mathrm{se}$ & z-ratio & Prob. \\
\hline Intercept & 0.448 & 0.557 & 0.804 & 0.422 \\
Condition & -0.171 & 0.832 & -0.206 & 0.837 \\
Block & 0.507 & 0.238 & 2.132 & 0.033 \\
Condition*Block & 0.290 & 0.384 & 0.756 & 0.450 \\
AIC & 194.130 & & & \\
& & & & \\
II Categories & $\mathrm{b}$ & $\mathrm{se}$ & z-ratio & Prob. \\
\hline Intercept & -1.935 & 0.623 & -3.105 & 0.002 \\
Condition & 0.057 & 0.827 & 0.069 & 0.945 \\
Block & 0.074 & 0.223 & 0.333 & 0.739 \\
Condition*Block & 0.307 & 0.291 & 1.055 & 0.291 \\
AIC & 255.95 & & & \\
\hline
\end{tabular}

For the RD categories, the ANOVA revealed a significant effect of Block indicating that Block was a significant predictor of whether or not participants adopted the optimal strategy, $X^{2}=12.90, d f=1, p=0.0003$. There was no significant effect of Condition, $X^{2}=1.039, d f=1, p=0.31$ and no significant interaction term assuming Block and Condition, $X^{2}=0.538, d f=1, p=0.44$. For the II categories, the ANOVA revealed a significant effect of Condition indicating that the experimental condition was 
was a significant predictor of whether or not participants adopted the optimal strategy, $X^{2}=7.531, d f=1, p=0.002$, but there was no significant effect of Block, $X^{2}=3.32, d f=$ $1, p=0.07$ and no significant interaction term assuming Block and Condition, $X^{2}=1.11$, $d f=1, p=0.29$.

\section{Discussion}

This experiment investigated a prediction of the COVIS theory of category learning. That is, whether asking participants to view and describe several example stimuli prior to category learning would facilitate the hypothesis testing process of the theorized explicit verbal learning system and that this would in turn facilitate category learning of RD categories but not II categories. Our data failed to find support for this hypothesis but we found an unexpected alternative pattern: that the prior verbal description tasks seemed to help participants learn the II categories by facilitating the transition to the optimal information integration strategy. The results of our exploratory analyses and model fitting offer support for the COVIS theory of category learning.

Though unexpected, our results are still consistent with COVIS category learning. Since the verbal system is assumed to have an initial bias in categorization, participants tend to first approach a categorization task using the verbal system and only switch to the nonverbal system when it is clear that the verbal system cannot produce good performance (Ashby et al., 1998; Minda \& Miles, 2010; Zeithamova \& Maddox, 2007). To make the transition, participants likely first exhaust the verbal system's hypothesis testing procedure by testing all of the possible rules. Describing the categorization stimuli in the pre-training session should have helped participants to learn that there 
were only two dimensions on which to base categorization rules. Participants in the Describe condition who were assigned to the II category may have been more likely to transition to the nonverbal system because they were able to exhaust the hypothesis testing process more quickly than participants in the Control condition. With the knowledge that there was a very limited set of possible categorization rules (i.e., one based on frequency, one based on orientation), participants in the II-Describe condition may have been able to efficiently test all plausible rules and transition to the nonverbal system. Participants in the Control condition would have taken longer to make this transition because it would have been less obvious to them when the hypothesis testing strategy should be abandoned. Our modelling analysis seems to have borne this out. Conversely, Filoteo, Lauritzen, and Maddox (2010) prolonged the hypothesis testing procedure by adding an irrelevant stimulus dimension, which increased the number of possible categorization rules. In their study this impeded II performance and decreased optimal strategy use. Together, this suggests that the duration of the hypothesis testing procedure is an important factor that affects the transition to the nonverbal system.

We initially expected that verbally describing categorization stimuli would improve the performance of the verbal system. However, describing the categorization stimuli had little, if any, effect on RD learning. Participants in the Describe condition did not perform significantly better or use an optimal frequency strategy any more than participants in the Control condition. It seems that prior knowledge of the stimuli, specifically the number of stimulus dimensions, may not be particularly helpful for learning these $\mathrm{RD}$ categories. That is not to say that stimulus knowledge in general is 
unimportant for the verbal system but it may be that prior knowledge confers little advantage in this case. One reason for this might be that the rule is straightforward and fairly easy for participants to find. Given the design of the RD stimuli in the current study, it is likely that most participants used frequency as the basis for either the first or second rule they tried. Therefore, in-depth stimulus knowledge would not be necessary to perform well on this RD category set; it is possible that a more complex RD category set could have benefitted from prior stimulus description.

Although there was no significant effect of stimulus description on RD learning, there was a relatively consistent $3 \%$ advantage for the Describe condition. This non-significant RD-Describe advantage was not much smaller than the 5-6\% II-Describe advantage, which was significant. Note that we are not making the claim that stimulus description was more beneficial for the nonverbal than the verbal system. Instead, the purpose of these exploratory analysis was to determine whether stimulus description was beneficial to the nonverbal system. Therefore, our conclusions do not rest heavily on whether the trend of increased RD performance was a significant one. Given that our exploratory hypotheses regarded the transition to the nonverbal system during II learning, it is important that we showed that II categorization performance and nonverbal strategy use were affected by our manipulation. Although this was not a predicted hypothesis, our exploratory analyses indicate an agreement with the COVIS theory of category learning.

We have characterized the verbal and nonverbal systems as being in competition with one another, with the categorization decisions based on the dominant system's 
response. This assumption comes from the most common description of the multiple system theory (Ashby et al., 1998; Ashby \& Valentin, 2017). It is conceivable that instead, the systems work in parallel and combine information to come to a decision. In practice, this would not likely produce a different pattern of categorization decision than a competition model because when the systems' responses are at odds, the strongest system would prevail. Our results do not distinguish between these two scenarios. Similarly, our results do not explicate how the shift between systems occurs. One possibility is an abrupt shift from one system to the other. Another possibility is that the systems are engaged probabilistically in proportion to their relative weights and the shift occurs gradually. Since our computational models are fit to entire blocks of data, we cannot determine the speed at which the transition between systems occurs.

We designed and interpreted the present study under the assumption of multiple category-learning systems, but instead, one could assume a single category-learning system that is able to learn RD and II category sets (see, e.g., (Newell, Dunn, \& Kalish, 2011). In one version of the single-system account, RD and II categories may be learned using a single category-learning system that implements different strategies to learn each type of category; a verbal strategy may be the default categorization approach, and the likelihood of engaging in a nonverbal strategy may be affected by characteristics of the task and learning environment. Strictly speaking, this could be a single-system account but has many similarities with the multiple-systems framework that we have assumed. Although there is convincing evidence that these strategies are subserved by distinct neurobiological systems (e.g., Maddox \& Filoteo, 2001; Nomura et al., 2007; 
Nomura \& Reber, 2012), this is not the focus of the present article, nor is it an especially important point for our findings. Instead, the important point is that there are multiple approaches for learning new categories and it is possible to modulate the use of each approach, regardless of whether they are construed as separate systems, strategies, or otherwise. On several occasions our lab has explored the notion that people rely on different strategies and core cognitive processes when learning different kinds of categories and that these distinction do not always cleanly delineate between a multipleor single-system approach (Minda \& Miles, 2010; Rabi, Miles, \& Minda, 2015). +

\section{Conclusions}

The findings of the current study are compatible with others that have manipulated the interaction between COVIS's hypothesized categorization systems by facilitating the transition to the nonverbal system. In our experiment, we showed the paradoxical effect that increasing the efficiency of the hypothesis testing procedure expedites the transition to the nonverbal system. This research is part of a growing body of work examining the interactions between categorization systems and provides new evidence about how control of the categorization process is shared between the systems. 


\section{References}

Ashby, F. G., Alfonso-Reese, L. A., Turken, A. U., \& Waldron, E. M. (1998). A neuropsychological theory of multiple systems in category learning. Psychological Review, 105(3), 442-481.

Ashby, F. G., \& Gott, R. E. (1988). Decision rules in the perception and categorization of multidimensional stimuli. Journal of Experimental Psychology. Learning, Memory, and Cognition, 14(1), 33-53.

Ashby, F. G., Maddox, W. T., \& Bohil, C. J. (2002). Observational versus feedback training in rule-based and information-integration category learning. Memory \& Cognition, 30(5), 666-677.

Ashby, F. G., \& Valentin, V. V. (2017). Chapter 7 - Multiple Systems of Perceptual Category Learning: Theory and Cognitive Tests. Handbook of Categorization in Cognitive Science (Second Edition), 157-188.

Eichenbaum, H. (1997). Declarative memory: insights from cognitive neurobiology. Annual Review of Psychology, 48, 547-572.

Evans, J. S. B. T. (2003). In two minds: dual-process accounts of reasoning. Trends in Cognitive Sciences, 7(10), 454-459.

Filoteo, J. V., Lauritzen, S., \& Maddox, W. T. (2010). Removing the frontal lobes: the effects of engaging executive functions on perceptual category learning. Psychological Science, 21(3), 415-423.

Filoteo, J. V., Maddox, W. T., Ing, A. D., \& Song, D. D. (2007). Characterizing rule-based 
category learning deficits in patients with Parkinson's disease. Neuropsychologia, 45(2), 305-320.

Gawronski, B., \& Bodenhausen, G. V. (2006). Associative and propositional processes in evaluation: an integrative review of implicit and explicit attitude change. Psychological Bulletin, 132(5), 692-731.

Maddox, W. T., \& Ashby, F. G. (1993). Comparing decision bound and exemplar models of categorization. Perception \& Psychophysics, 53(1), 49-70.

Maddox, W. T., Ashby, F. G., \& Bohil, C. J. (2003). Delayed feedback effects on rule-based and information-integration category learning. Journal of Experimental Psychology. Learning, Memory, and Cognition, 29(4), 650-662.

Maddox, W. T., Bohil, C. J., \& Ing, A. D. (2004). Evidence for a procedural-learning-based system in perceptual category learning. Psychonomic Bulletin \& Review, 11(5), 945-952.

Maddox, W. T., \& Filoteo, J. V. (2001). Striatal contributions to category learning: quantitative modeling of simple linear and complex nonlinear rule learning in patients with Parkinson's disease. Journal of the International Neuropsychological Society: JINS, 7(6), 710-727.

Maddox, W. T., Filoteo, J. V., Hejl, K. D., \& Ing, A. D. (2004). Category number impacts rule-based but not information-integration category learning: further evidence for dissociable category-learning systems. Journal of Experimental Psychology. Learning, Memory, and Cognition, 30(1), 227-245.

Maddox, W. T., Filoteo, J. V., \& Lauritzen, J. S. (2007). Within-category discontinuity 
interacts with verbal rule complexity in perceptual category learning. Journal of Experimental Psychology. Learning, Memory, and Cognition, 33(1), 197.

Maddox, W. T., \& Ing, A. D. (2005). Delayed feedback disrupts the procedural-learning system but not the hypothesis-testing system in perceptual category learning. Journal of Experimental Psychology. Learning, Memory, and Cognition, 31(1), $100-107$.

Markman, A. B., Maddox, W. T., \& Worthy, D. A. (2006). Choking and excelling under pressure. Psychological Science, 17(11), 944-948.

Miles, S. J., \& Minda, J. P. (2011). The effects of concurrent verbal and visual tasks on category learning. Journal of Experimental Psychology. Learning, Memory, and Cognition, 37(3), 588-607.

Minda, J. P., Desroches, A. S., \& Church, B. A. (2008). Learning rule-described and non-rule-described categories: a comparison of children and adults. Journal of Experimental Psychology. Learning, Memory, and Cognition, 34(6), 1518-1533.

Minda, J. P., \& Miles, S. J. (2010). The influence of verbal and nonverbal processing on category learning. In B. H. Ross (Ed.), Psychology of Learning and Motivation (Vol. 52, pp. 117-162). Academic Press.

Newell, B. R., Dunn, J. C., \& Kalish, M. (2011). Systems of category learning: fact or fantasy? In Psychology of learning and motivation (Vol. 54, pp. 167-215). Elsevier.

Nomura, E. M., Maddox, W. T., Filoteo, J. V., Ing, A. D., Gitelman, D. R., Parrish, T. B., ... Reber, P. J. (2007). Neural correlates of rule-based and information-integration visual category learning. Cerebral Cortex , 17(1), 37-43. 
Nomura, E. M., \& Reber, P. J. (2012). Combining computational modeling and neuroimaging to examine multiple category learning systems in the brain. Brain Sciences, 2(2), 176-202.

Nosofsky, R. M., Stanton, R. D., \& Zaki, S. R. (2005). Procedural interference in perceptual classification: implicit learning or cognitive complexity? Memory \& Cognition, 33(7), 1256-1271.

Rabi, R., Miles, S. J., \& Minda, J. P. (2015). Learning categories via rules and similarity: comparing adults and children. Journal of Experimental Child Psychology, 131, 149-169.

Rabi, R., \& Minda, J. P. (2016). Category learning in older adulthood: A study of the Shepard, Hovland, and Jenkins (1961) tasks. Psychology and Aging, 31(2), 185-197.

Rabi, R., \& Minda, J. P. (2017). Familiarization may minimize age-related declines in rule-based category learning. Psychology and Aging, 32(7), 654-674.

R Core Team, \& Others. (2008). R: A language and environment for statistical computing. R foundation for statistical computing Vienna, Austria.

Sloman, S. A. (1996). The empirical case for two systems of reasoning. Psychological Bulletin, 119(1), 3-22.

Smith, E. E., \& Grossman, M. (2008). Multiple systems of category learning. Neuroscience and Biobehavioral Reviews, 32(2), 249-264.

Waldron, E. M., \& Ashby, F. G. (2001). The effects of concurrent task interference on category learning: evidence for multiple category learning systems. Psychonomic Bulletin \& Review, 8(1), 168-176. 
Worthy, D. A., Maddox, W. T., \& Markman, A. B. (2009). Less is more: Stimulus-feedback co-occurrence in perceptual category learning. Proceedings of the Cognitive Science Society, 6.

Zeithamova, D., \& Maddox, W. T. (2006). Dual-task interference in perceptual category learning. Memory \& Cognition, 34(2), 387-398.

Zeithamova, D., \& Maddox, W. T. (2007). The role of visuospatial and verbal working memory in perceptual category learning. Memory \& Cognition, 35(6), 1380-1398. 


\section{Appendix A}

For the model-based strategy analysis, we adopted the assumptions and model-based analysis methods of GRT. First, we assumed that a participant categorizes stimuli by partitioning his perceptual space into two regions. This partition is known as a decision bound. The participant categorizes each stimulus according to the region in which it falls. That is, given a stimulus i and the decision bound function $h_{x_{i}}$, the categorization decision can be described as "Respond A if $h_{x_{i}}>0$, and respond B otherwise".

Second, on the basis of GRT, we assumed that perception of a stimulus is subject to noise (i.e., perceptual noise; ep), so that repeated exposures of the same stimulus do not always yield the same perceptual effect. The perceived coordinate of the stimulus $i$ is denoted as xi, and its distribution is multivariate normal. This assumption makes the response to a stimulus probabilistic. The probability of responding A to xi is

$$
P\left(x_{i}\right)=P\left[x_{i}\right] .
$$

In the present application, the decision bounds are assumed to be linear and can be written as:

$$
h\left(x_{i}\right)=b^{T} x_{i}+c+\in,
$$


where $\mathrm{b}$ represents a vector of constants and $\mathrm{c}$ represents some scalar. The variable denotes random noise with zero mean and variance $\sigma^{2}$, representing criterial noise (i.e., noise in the participant's memory of the decision bound). Thus, the mean and variance of $h(x i)$ are

$$
b^{T} x_{i}+c \text { and } b^{T} \Sigma b+\sigma_{c}^{2},
$$

respectively.

Because the distribution of xi is multivariate normal and the distribution of ec is assumed to be normal, the probability of responding A to xi becomes

$$
P(x)=\phi\left(\frac{b^{T} x_{i}+c}{\sqrt{b^{T} \Sigma b+\sigma^{2}}}\right)
$$

where $\Phi(\cdot)$ is the cumulative distribution function of the standard normal distribution.

With these assumptions in place, we attempted to find the configuration of the decision bound that best describes each participant's categorization behavior in each block of trials. The stimuli in the present study can be represented in two dimensions - that is, frequency and orientation. Different configurations of the decision bound can be interpreted as different categorization strategies. In particular, when the slope of the decision bound is zero or undefined, the participant attended to only one of the dimensions for categorization. When the slope is defined and nonzero, the participant attended to and integrated the information from both dimensions. By changing the number of parameters that can vary freely, we can fit several different 
decision bound models that correspond to three classes of strategies (frequency, orientation, II).

One frequency strategies was fit to participants' responses in which the intercept was set to the optimal value, and the noise parameter was allowed to vary. One corresponding orientation strategies with set intercept was also fit. One II strategy was fit in which the noise parameter was allowed to vary, and the slope and intercept were set to optimal values.

In addition to the decision-bound-based strategies, we also fit a guessing model where the classification decision is based on random guessing. This model assumed that participants randomly responded A or B with an equal probability for each response (i.e., $P\left(x_{i}\right)=P\left(x_{i}\right)=.5$ ). This model had no free parameters.

The free parameters for each model were estimated by the maximum likelihood method. The likelihood of observing a given set of response patterns, $r=\left(r_{1}, \ldots, r_{n}\right)$, is

$$
L(r)=\prod_{i=1}^{n} P\left(x_{i}\right)^{r_{i}} * P\left(B \mid x_{i}\right)^{1-r_{i}},
$$

where $\mathrm{ri}=1$ if response $\mathrm{A}$ was made to $\mathrm{xi}$ and $\mathrm{ri}=\mathrm{o}$ if response $\mathrm{B}$ was made. The relative fit of the models was compared using AIC:

$$
B I C=-2 k-2 \operatorname{Ln}(L)
$$


where $\mathrm{df}$ is the number of free parameters in the model and $\mathrm{n}$ is the number of trials inputted into the model. AIC is a measure of goodness of fit that takes into account the number of free parameters in the model. Because AIC is a measure of goodness of fit, small values indicate a good fit of the strategy to the data. 


\section{Author Note}

Sarah J. Miles, Bailey Brashears, Joshua Hatherley and John Paul Minda are all at the Department of Psychology, The University of Western Ontario. This research was supported by an NSERC Discovery Grant to JPM. These experiments were completed in partial fulfillment of SJM's doctoral dissertation. Correspondences should be addressed to John Paul Minda at the Department of Psychology, The University of Western Ontario, London, ON, N62 5C2, CANADA, or by email at jpminda@uwo.ca. 